\title{
The multi-PMT optical module for the IceCube-Upgrade
}

\author{
Lew Classen $^{1, *}$ and Alexander Kappes ${ }^{1, * *}$ for the IceCube Collaboration \\ ${ }^{1}$ Institut für Kernphysik, Westfälische Wilhelms-Universität Münster, 48149 Münster, Germany
}

\begin{abstract}
Following the first observation of an astrophysical high-energy neutrino flux with the IceCube Neutrino Observatory in 2013, planning for an upgrade of the detector is progressing, which will expand the capabilities of the detector both at low and high neutrino energies. A substantial contribution to the improved performance is anticipated to be achieved by the application of advanced optical module technology. The multi-PMT optical module, mDOM, consists of 24 3-inch PMTs which provide, amongst others, a large, homogeneous photosensitive area and sensitivity to the incident direction of photons. After an introduction, the current status of the mDOM development is presented with emphasis on the characterization of the photomultipliers under consideration.
\end{abstract}

\section{The multi-PMT optical module (mDOM)}

Located in the deep glacial ice of Antarctica, IceCube [1] is the world's largest neutrino telescope. Originally designed for the investigation of the neutrino sky on the TeV to $\mathrm{PeV}$ energy scale and beyond, the energy threshold was lowered to $\sim 10 \mathrm{GeV}$ by the DeepCore [2] extension. In a next step, IceCube's potential will be further enhanced through the installation of seven new strings in the DeepCore region (IceCube-Upgrade) which will be equipped with up to 120 optical modules each. This will lower IceCube's energy threshold to few $\mathrm{GeV}$ and provide a platform for improved calibration of the detector. Novel optical sensors will have a key role in the expected performance enhancements. Among the new designs is the so-called mDOM, a multi-PMT Digital Optical Module. Optical modules based on this concept, first used for deep-sea detectors by the KM3NeT Collaboration [3], feature an array of several small photomultipliers (PMTs) housed inside a transparent pressure vessel (see Fig. 1). Currently, the designs of mechanical and electronic components of the mDOM are being finalized and a first performance demonstrator module is under construction.

\section{2 mDOM components}

\subsection{Photomultipliers (PMTs)}

With the $\mathrm{mDOM}$ being based on the KM3NeT multi-PMT module, the baseline PMT is a derivative of the well-characterized KM3NeT model from Hamamatsu. The mDOM however introduces stricter spacial constraints: the PMT including the base is limited to a conical volume with an opening angle of $45^{\circ}$ and an edge length of $\sim 160 \mathrm{~mm}$.

\footnotetext{
*e-mail: lew.classen@wwu.de

**e-mail: alexander.kappes@uni-muenster.de
} 

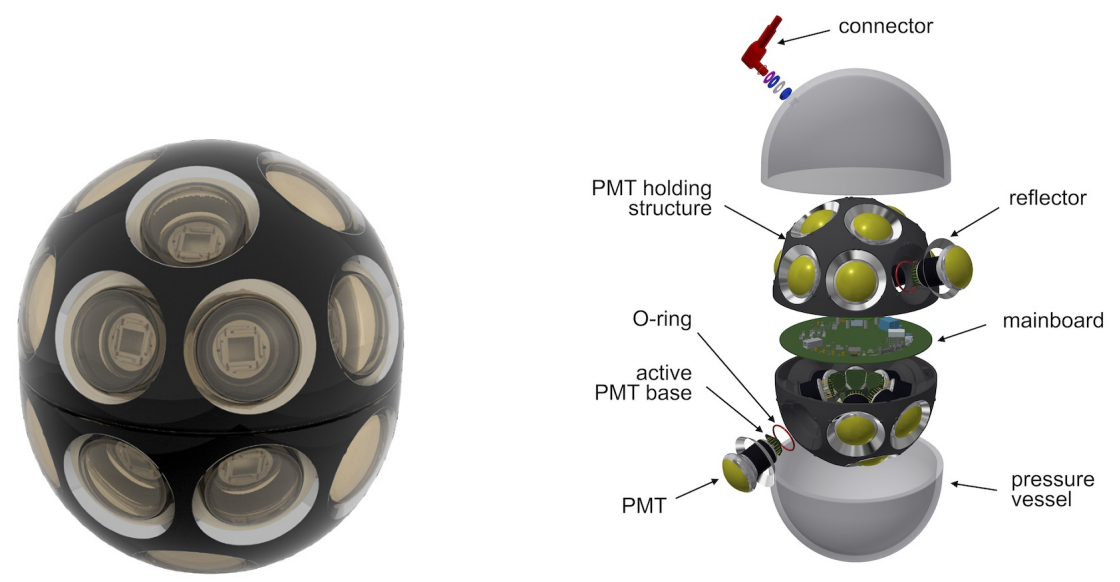

Figure 1. mDOM overview: Left: Rendered view. Right: Exploded view.
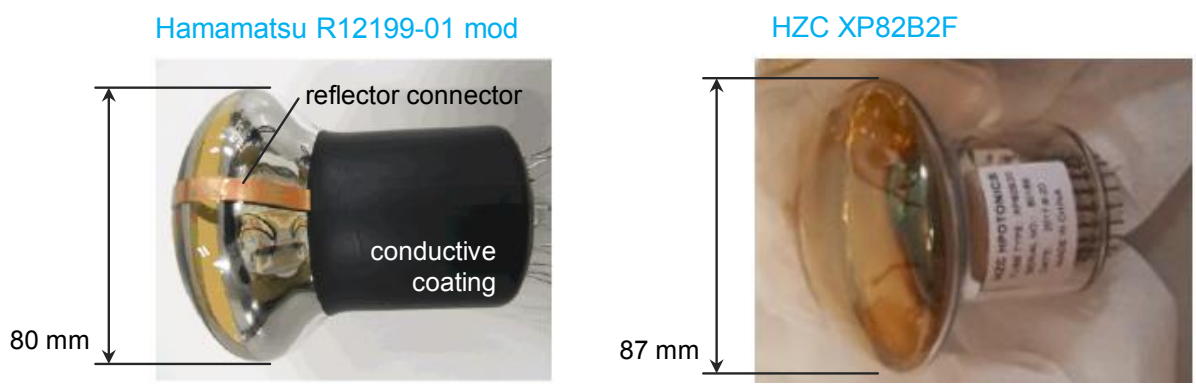

Figure 2. Candidate PMTs for the multi-PMT Digital Optical Module (mDOM).

The Hamamatsu type R12199-01 MOD HA PMT (see Fig. 2, left) is a $80 \mathrm{~mm}$ photomultiplier based on the KM3NeT model R12199-02. It was modified for the use in low-background applications with tight spacial constraints such as the mDOM. To fit this usage profile, the PMT features a tube that has been reduced in length from $97 \mathrm{~mm}$ to $93 \mathrm{~mm}$ and a so-called "HA-coating", a conductive layer located on the outside surface of the tube surrounding the electron multiplier system which is electrically connected to the photocathode. This feature is designed to reduce and stabilize the dark-noise rate for PMTs operated at negative HV. In addition, the latest modification features a dedicated copper strip for easy connection of the optical reflectors (see Section 2.4) to photocathode potential. This measure is also directed at minimizing noise as conductive objects at non-photocathode potential close to the photocathode tend to invoke high and unstable dark-noise rates [4].

A promising alternative PMT candidate is the XP 82B2F PMT manufactured by HZC photonics (see Fig. 2, right). The device fits the tight spatial constraints of the $\mathrm{mDOM}$ while featuring a larger tube diameter of $87 \mathrm{~mm}$. Being pin-compatible to the baseline Hamamatsu model, the integration effort is expected to be moderate while the larger tube diameter provides an increase in overall module photo-sensitive area of $\sim 20-30 \%$. 


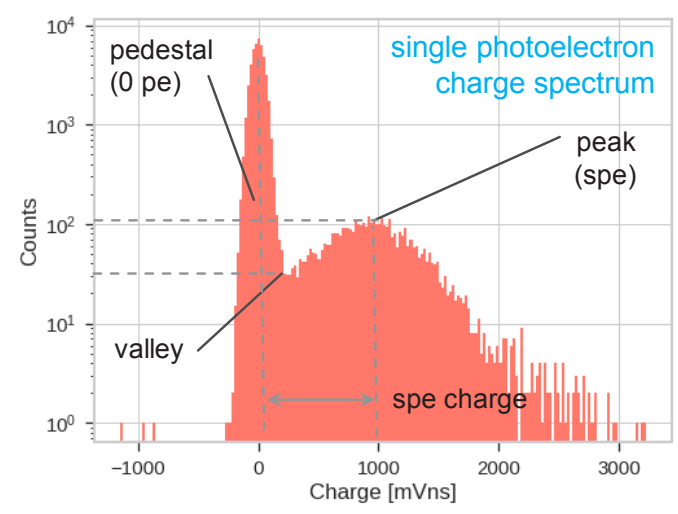

Figure 3. Single photoelectron (SPE) charge spectrum. Features used for gain and peak-to-valley ratio determination are highlighted.

\subsection{Photomultiplier performance}

PMT testing was carried out with slightly different setups in Erlangen (Hamamatsu PMT) and Madison (HZC PMT). 66 Hamamatsu PMTs were tested while the HZC batch consisted of three tubes. The respective results are discussed in the following paragraphs. The performance of the KM3NeT PMT R12199-02 [5], the progenitor of the tested Hamamatsu model, was considered as reference.

\section{Gain}

The gain $g$ was derived from a single photoelectron (SPE) charge spectrum (illustrated in Fig. 3) as $g=\Delta Q / e$, where $\Delta Q$ is the position of the single-photoelectron charge peak relative to the pedestal determined from a fit of the distribution based on [6], and $e$ is the electron charge. For both PMT models the gain was determined in relation to the PMT supply voltage (also known as gain slope). This information allowed to identify the voltage corresponding to the nominal gain (Erlangen setup: $5 \cdot 10^{6}{ }^{1}$; Madison setup: $1 \cdot 10^{72}$ ) at which the PMTs were operated for the rest of the study. In both cases the supply voltages were well between $900 \mathrm{~V}$ and $1300 \mathrm{~V}$, the range of the reference model [5]. Details are presented in Fig. 4.

\section{Peak-to-valley ratio}

The peak-to-valley ratio, a quality parameter combining the baseline noise and SPE resolution, was also derived from the above-mentioned SPE spectrum (Fig. 3). Results for the Hamamatsu and HZC tubes are presented in Fig. 5. As large peak-to-valley ratios are favorable, the HZC model features a slightly superior performance. However, the comparability is somewhat limited as the parameter increases with gain.

\section{Transit-time spread}

The transit-time spread (TTS) or signal jitter defines the single-photon time resolution achievable with a PMT. It was measured in terms of the standard deviation of a Gaussian distribution fitted to the main peak of the arrival time distribution. The latter was recorded illuminating the PMT with a sub-nanosecond pulser and a mean photon illumination level $<0.1$ pe. The results are displayed in Fig. 6. The Hamamatsu PMT is slightly superior to the HZC model. This difference is expected to become more pronounced if both PMTs are operated at identical gain. The performace of both PMTs is comparable to the reference model [5].

\footnotetext{
${ }^{1}$ The gain envisioned for the $\mathrm{mDOM}$.

${ }^{2}$ The gain of the current IceCube optical modules.
} 

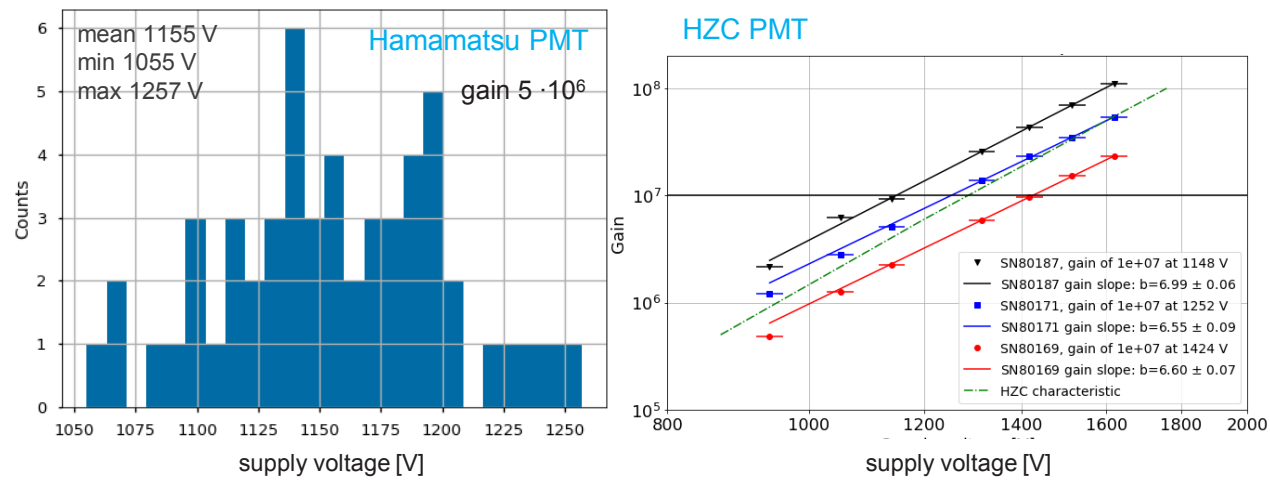

Figure 4. Left: Distribution of nominal PMT supply voltages, i.e. the voltages needed to operate the PMTs at the standard gain of $5 \cdot 10^{6}$, for the tested Hamamatsu PMTs. Right: Gainslopes for the HZC PMTs.

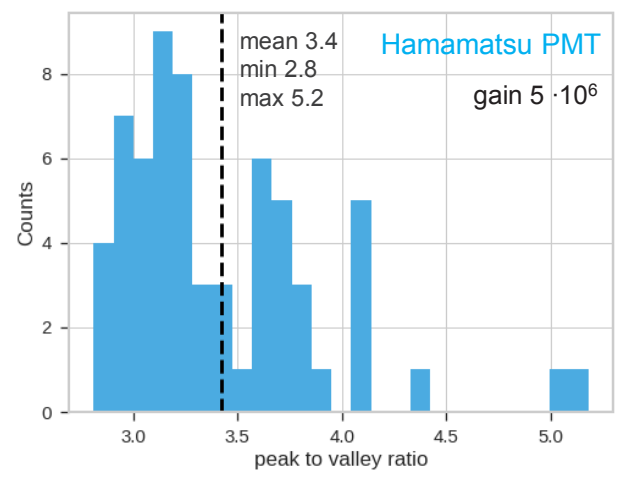

\begin{tabular}{cc} 
HZC PMT & gain $10^{7}$ \\
\hline PMT & peak-to-valley ratio \\
\hline SN80187 & 3.1 \\
SN80171 & 4.3 \\
SN80169 & 3.6 \\
\hline
\end{tabular}

Figure 5. Left: Peak-to-valley ratio distribution obtained for 66 Hamamatsu PMTs at a gain of $5 \cdot 10^{6}$. Right: Peak-to-valley ratios for the tested HZC PMTs at a gain of $10^{7}$.

\section{Dark noise rate}

As the deep ice at the South Pole has negligible optical activity the dependence of the darkrate on temperature is of particular interest. The test sample consisted of two Hamamatsu PMTs and one specimen of the HZC model. The results are illustrated in Fig. 7. Towards lower temperature, the rate first decreases due to reduced thermionic emission from the photocathode. Below $0{ }^{\circ} \mathrm{C}$, light from radioactive decays in the glass of the PMT envelop becomes the dominant source of noise. In the temperature range most relevant for the IceCubeUpgrade (around $-20^{\circ} \mathrm{C}$ at the installation depths of the modules [7]) the rate for both PMT models is well below $100 \mathrm{~s}^{-1}$. The larger dark rate of the HZC model is at least partially caused by the larger photocathode area and the higher operation voltage associated with the higher gain selected (the shorter dead-time of $0.5 \mu$ s during data taking for the HZC PMT compared to $1 \mu$ s for the Hamamatsu PMT accounts only for few counts per second). 


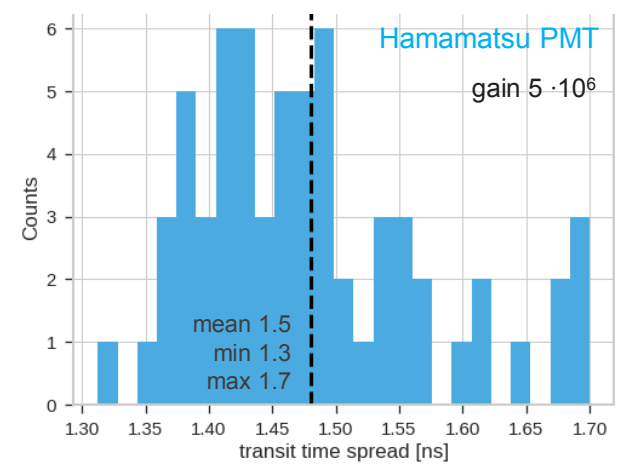

\begin{tabular}{cc} 
HZC PMT & gain $10^{7}$ \\
\hline PMT & TTS [ns] \\
\hline SN80187 & 1.8 \\
SN80171 & 1.8 \\
SN80169 & 1.4 \\
\hline
\end{tabular}

Figure 6. Transit-time spread (TTS, $\sigma$ ) for the tested Hamamatsu (left) and HZC (right) PMTs, obtained at respective standard gains.
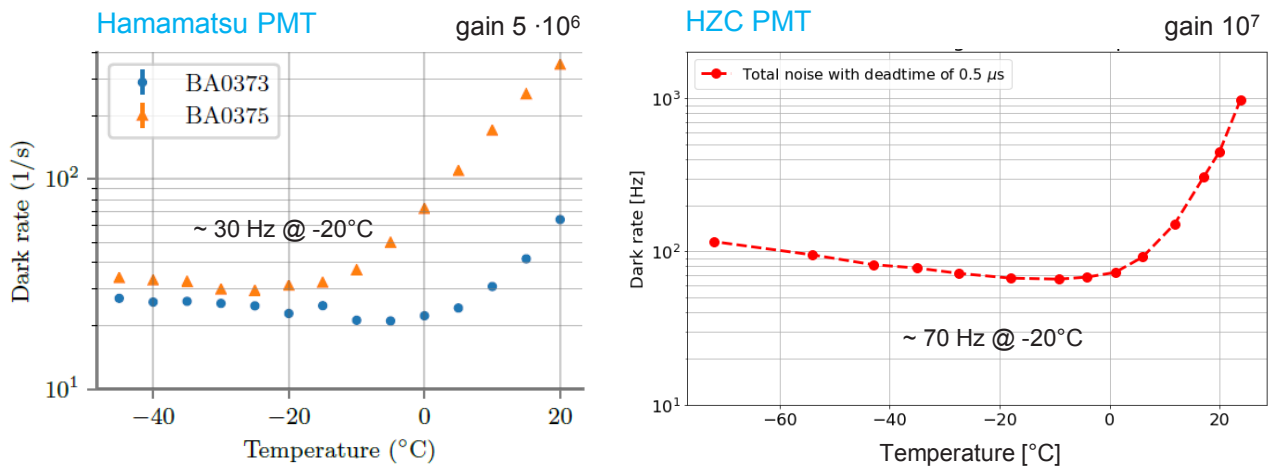

Figure 7. Dark rate as a function of the ambient temperature, measured for two specimen of the Hamamatsu model (left) with a dead-time of $1 \mu$ s and one HZC PMT (right) with a dead-time of $0.5 \mu \mathrm{s}$. The PMTs were operated at respective standard gains at all temperatures.

\section{Quantum efficiency}

As of now, the quantum efficiency was measured for the Hamamatsu model only. Monochromatic light selected via a monochromator from the spectrum of a continuous light source was guided onto the PMT's photocathode, illuminating the innermost region of approximately $1 \mathrm{~cm}^{2}$. The resulting photocathode current was collected via a dedicated base shorting the electron multiplier system and calibrated with the current from a reference photo-diode exposed to an identical photon flux. The resulting quantum efficiency spectra are presented in Fig. 8. The values are comparable to measurements by the manufacturer as well as to the quantum efficiency of the reference model [5].

\section{Response uniformity}

For one specimen of the Hamamatsu PMT the response uniformity was investigated in terms of the relative anode signal. The output of a pulsed light source $(\lambda=390 \mathrm{~nm})$ was fed into a fiber. The collimated fiber output, oriented parallel to the PMT axis (beam diameter $<1 \mathrm{~mm}$ ), 

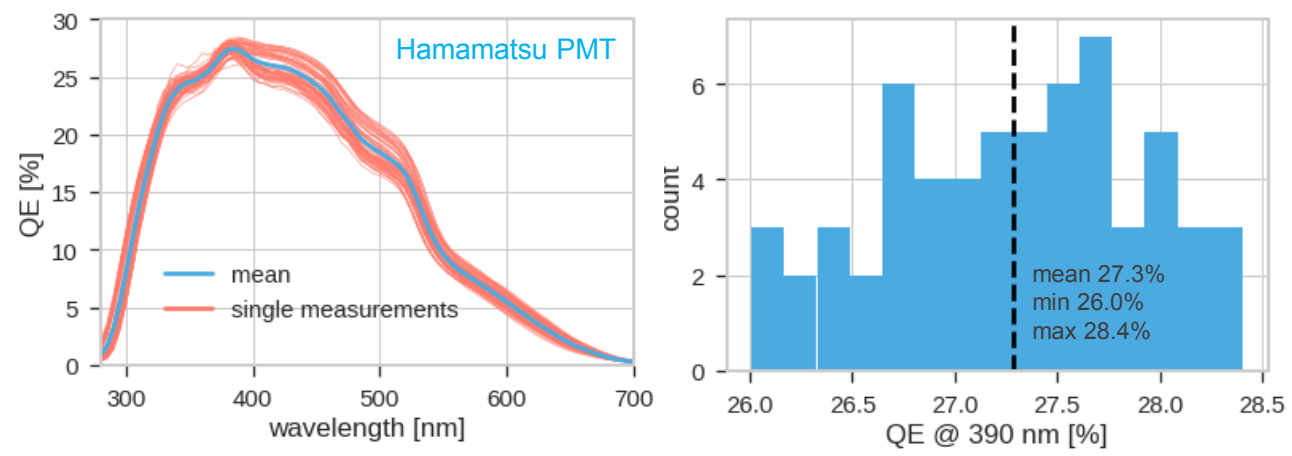

Figure 8. Left: Quantum efficiency (QE) spectra for all tested Hamamatsu PMTs. Left: Distribution of the quantum efficiency for $390 \mathrm{~nm}$ photons.
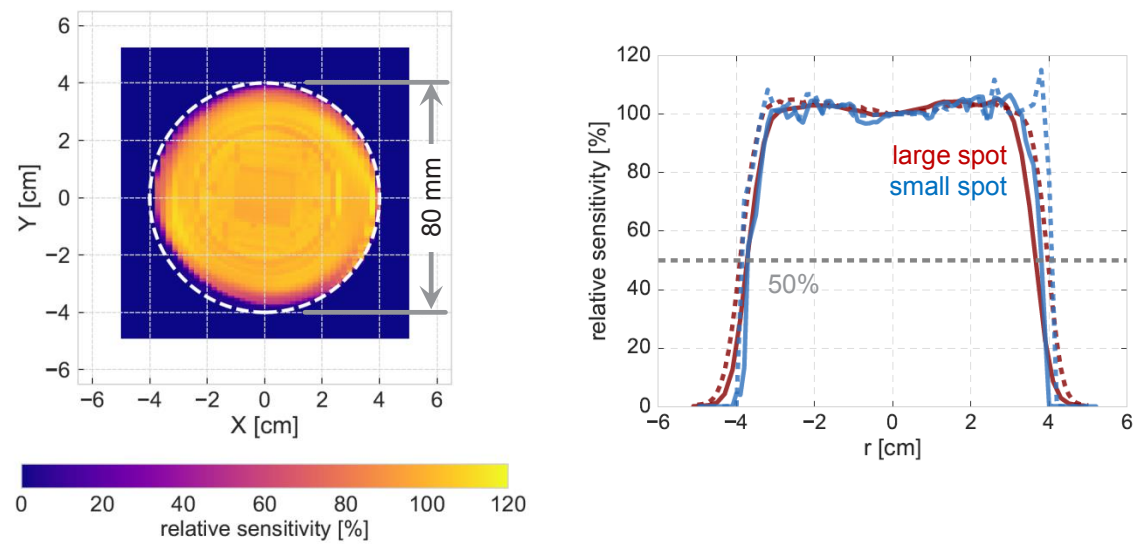

Figure 9. Left: Anode response scan of the Hamamatsu R12199-02. The dashed white line marks a circle with $80 \mathrm{~mm}$ diameter. Values are normalized to the center. Right: Example vertical and horizontal slices selected from the scans, comparing the results obtained with different beam diameters.

was moved in $x-y$ across the entrance window of the PMT determining the photo-current at each position. Results of the scan, presented in Fig. 9, show the contribution of internal reflections inside the PMT. The data also allowed to derive an effective diameter for the PMT under investigation (integration of the area weighted by the relative signal strength) which was found to be $77 \mathrm{~mm}$ to $78 \mathrm{~mm}$, exceeding the more conservative statement of $72 \mathrm{~mm}$ by the manufacturer.

\subsection{Readout electronics and high voltage generation}

The general requirements for the readout and high voltage systems are a low power consumption, low sensitivity to interference signals, a low footprint and high reliability, paired with the ability to sample semi-complex wave-forms from the PMTs. IceCube uses a modular architecture of common electronics components (communication, timing etc.) with well-defined 


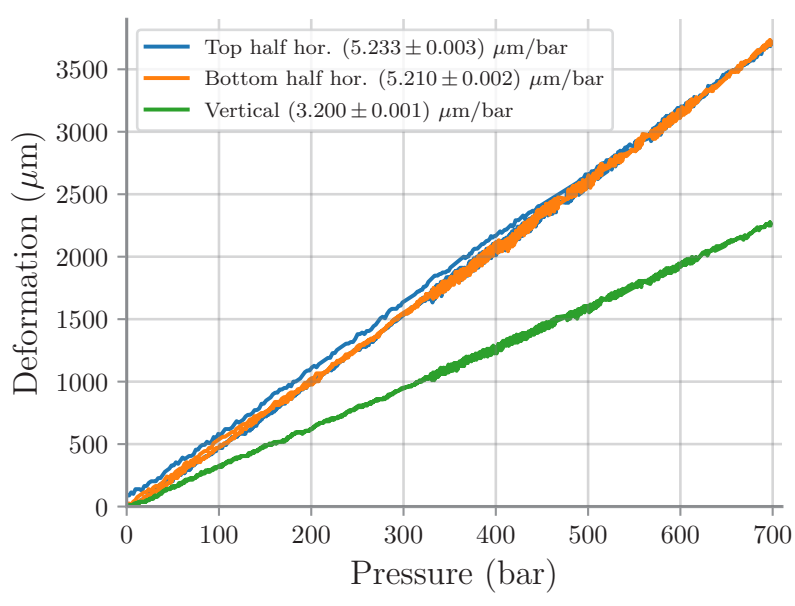

Figure 10. Horizontal and vertical (green line) shrinkage of an $\mathrm{mDOM}$ pressure vessel as function of external pressure in a hyperbaric chamber. The horizontal deformation was measured separately in the upper (blue line, partially hidden) and lower (orange line) vessel half.

interfaces to reduce the development efforts for the different modules. The baseline of the high-voltage system is a low-power Cockcroft-Walton circuitry for high-voltage generation on the PMT base. The baseline design for the readout consists of "slow" ADCs (e.g. 12 bit, 100 MSPS) which digitize the signals individually for each PMT. Precise leading-edge timing is provided by fast sampling ( $>500 \mathrm{MHz}$ ) of a comparator output which analyzes the analog PMT signal at a low threshold of about 0.2 pe.

\subsection{Support structure and reflectors}

A support structure is used to position PMTs, reflectors and the main electronics board inside the pressure vessel. This component is produced via selective laser sintering (a form of 3D printing) from white polyamide and subsequently died black with non-conductive color. The color was chosen as a white structure only marginally increases the overall module sensitivity while enhancing the amount of undesired photon scattering. In addition to positioning, the design ensures electric insulation of the photocathode at negative HV with gel pockets.

Mounted around the entrance window of the PMTs, light concentrators or reflectors increase the PMT's effective area for vertical illumination. The devices are produced from enhanced-reflectivity coated aluminum sheets. The reflectors allow to reclaim photons otherwise lost due to shadowing and absorption in glass and gel. With respect to realistic configurations the utilization of reflectors yields an extra $\sim 20 \%$ in overall module sensitivity. The material was chosen to maximize the average Cherenkov-weighted reflectivity based on lab measurements. In a Monte Carlo study, simulating the incidence of a plane wave-front, the opening angle was optimized to provide maximum sensitivity for vertical photons, narrowing the PMT's field of view. This resulted in a final angle of $51^{\circ}$ with respect to the symmetry axis of the PMT. The effective concentration of the photon acceptance at small incidence angles comes at only marginal cost in terms of overall sensitivity $(<1 \%$ deviation from the achievable maximum).

\subsection{Pressure vessel and optical gel}

With its cylindrical extension around the equator (see Fig. 1), the mDOM pressure vessel slightly deviates from the usual spherical form factor. The design is driven by the limited diameter of the borehole and the non-reducible length of the PMTs. The pressure vessel is rated for 700 bar. This criterion, which is substantially larger than the hydrostatic pressure 
at the location of the detector, is based on the measured pressure peaks during re-freezing where up to 550 bar were observed during IceCube construction. The pressure rating has been verified in pressure tests: Figure 10 shows the reduction in horizontal and vertical diameter of the pressure vessel as function of external pressure. The shrinkage is reversible and agrees well with calculations based on finite element simulations.

Curing two-component silicone gel, poured between the PMTs and the pressure vessel, provides optical coupling as well as structural stability an cushioning. The baseline choice for prototyping is QGel 900 by Quantum Silicones which has been employed in the original IceCube optical module and proven to be usable at low ambient temperatures.

\section{Conclusion \& Outlook}

The multi-PMT Digital Optical Module (mDOM) is being developed for deployment in the deep ice at the South Pole for future IceCube extensions (IceCube-Upgrade, IceCube-Gen2 [8]). Harsh environmental conditions and available infrastructure pose stringent limits on the module parameters like size, power consumption and reliability. The mechanical design of the mDOM is well advanced and is currently being scrutinized and optimized. The Hamamatsu R12199-01 HA mod PMT, a well-characterized model suited for the application, is considered the baseline model for the $\mathrm{mDOM}$ and will be used in the construction of a demonstrator module. The HZC XP 82B2F could be a viable alternative: The examined PMTs feature a performance comparable to the Hamamatsu model and a larger batch has been ordered for further testing. These tests will also include the parameters not investigated so far, such as the quantum efficiency and the effective diameter, which will allow to assess the actual sensitivity gain from the utilization of the new model. For the readout and HV electronics baseline designs have been developed and prototypes are under construction. A fully integrated and operational module is expected to be available by the end of 2019 .

\section{References}

[1] M.G. Aartsen et al. (IceCube), JINST 12, P03012 (2017), arXiv: 1612.05093

[2] R. Abbasi et al. (IceCube), Astroparticle Phys. 35, 615 (2012), arXiv: 1109.6096

[3] H. Löhner et al. (KM3NeT), Nucl. Inst. Meth. A718, 513 (2013)

[4] Photomultiplier Tubes - Basics and Applications, Hamamatsu Photonics K.K., 3rd edn. (2007), https://www.hamamatsu.com/resources/pdf/etd/PMT_handbook_ v3aE.pdf

[5] S. Aiello et al., JINST 13, P05035 (2018)

[6] E. Bellamy et al., Nucl. Inst. Meth. A339, 468 (1994)

[7] M. Aartsen et al. (IceCube), Nucl. Inst. Meth. A711, 73 (2013), arXiv: 1301 . 5361

[8] M.G. Aartsen et al. (IceCube) (2014), arXiv : 1412 . 5106 